\title{
Nonabelian Groups with $(96,20,4)$ Difference Sets
}

\author{
Omar A. AbuGhneim \\ Department of Mathematics \\ University of Jordan \\ Amman, Jordan \\ o.abughneim@ju.edu.jo \\ Ken W. Smith \\ Department of Mathematics \\ Central Michigan University \\ Mt. Pleasant, MI 48859 \\ ken.w.smith@cmich.edu
}

Submitted: Apr 10, 2006; Accepted: Oct 10, 2006; Published: Jan 3, 2007

AMS Subject Classification: 05B10

\begin{abstract}
We resolve the existence problem of $(96,20,4)$ difference sets in 211 of 231 groups of order 96 . If $G$ is a group of order 96 with normal subgroups of orders 3 and 4 then by first computing 32- and 24-factor images of a hypothetical $(96,20,4)$ difference set in $G$ we are able to either construct a difference set or show a difference set does not exist.

Of the 231 groups of order 96, 90 groups admit $(96,20,4)$ difference sets and 121 do not. The ninety groups with difference sets provide many genuinely nonabelian difference sets. Seven of these groups have exponent 24 .

These difference sets provide at least 37 nonisomorphic symmetric $(96,20,4)$ designs.
\end{abstract}

\section{Introduction}

A $(v, k, \lambda)$ difference set is a subset $D$ of size $k$ in a group $G$ of order $v$ with the property that for every nonidentity $g$ in $G$, there are exactly $\lambda$ ordered pairs $(x, y) \in D \times D$ such that

$$
x y^{-1}=g .
$$

One may identify the set $D$ with an element $\hat{D}$ in the group $\operatorname{ring} \mathbb{Z}(G)$. In this case write

$$
\hat{D}=\sum_{g \in D} g
$$


and

$$
\hat{D}^{(-1)}=\sum_{g \in D} g^{-1}
$$

We also write $\hat{G}$ for $\sum_{g \in G} g$. D is a difference set if the group ring element $\hat{D}$ satisfies the equation

$$
\hat{D} \hat{D}^{(-1)}=(k-\lambda) 1_{G}+\lambda \hat{G} .
$$

If a group $G$ has a difference set $D$ then $\{g D: g \in G\}$ is the set of blocks of a symmetric $(v, k, \lambda)$ design with point set $G$. On this design $G$ acts by left multiplication as a sharply transitive automorphism group. Conversely, any symmetric design with a sharply transitive automorphism group on points is isomorphic to a design constructed from the set of left translates of a difference set. A difference set is said to be "genuinely nonabelian" if the underlying design has no abelian group acting regularly on points.

Difference sets with parameters $\left(q^{d+1}\left(\frac{q^{d+1}-1}{q-1}+1\right), q^{d} \frac{q^{d+1}-1}{q-1}, q^{d} \frac{q^{d}-1}{q-1}\right)$, where $q=p^{f}$ is a prime power, are known as McFarland difference sets. For $q=4$ and $d=1$, we obtain the $(96,20,4)$ parameters. For more details on symmetric designs and difference sets, the reader may consult [6], [11], [12], [13]. For more further discussion on McFarland difference sets, see [7], [14].

We say $D_{1} \in \mathbb{Z}(G), D_{2} \in \mathbb{Z}(G)$ are equivalent if there is an element $g \in G$ and an automorphism $\varphi$ of $G$ such that $D_{1}=g \varphi\left(D_{2}\right)$. We say that two difference sets are inequivalent if either they are subsets of nonisomorphic groups or if they are subsets in a common group $G$ but are not equivalent in $G$ (as defined above.) Inequivalent difference sets may give rise to isomorphic designs.

The adjacency matrix of a symmetric design has a set of invariant factors (or "elementary divisors", associated with the Smith Normal Form of the matrix.) If $A$ is the adjacency matrix of the design, there are invertible matrices $P$ and $Q$ such that $P A Q$ is a diagonal matrix. $P$ and $Q$ may be chosen so that the $(i, i)$ entry of $P A Q$ divides the $(i+1, i+1)$ entry of $P A Q$ (for all $i, 1 \leq i \leq v-1$.) In the special case of the parameters $(96,20,4)$, we may assume that $P A Q$ is the direct sum of six scalar matrices:

$$
P A Q=I_{r_{0}} \oplus 2 I_{r_{1}} \oplus 4 I_{r_{2}} \oplus 8 I_{r_{3}} \oplus 16 I_{r_{4}} \oplus 80 I_{r_{5}} .
$$

Since the prime 5 divides $k=20$ but does not divide $k-\lambda=16$, then the rank of $A$ over $G F(5)$ is 95 and $r_{5}=1$. Thus the sum of the other matrix sizes, $r_{0}+r_{1}+r_{2}+r_{3}+r_{4}$, is 95. The rank of $A$ over $G F(2)$ is $r_{0}$, the size of the identity matrix in this direct sum. We will abbreviate the list of invariant factors with the 5 -tuple $\left(r_{0}, r_{1}, r_{2}, r_{3}, r_{4}\right)$. For example, there are at least two distinct difference sets in the elementary abelian group $\operatorname{GAP}[96,231]$; one has invariant factor abbreviated by $(30,2,32,2,29)$ while the other has invariant factor abbreviated by $(30,1,34,1,29)$. Both have 2-rank equal to 30. Although nonisomorphic designs may have the same invariant factors, if the designs have different invariant factors, they must be nonisomorphic. We found 37 different patterns of invariant factors in the exhaustive search and so have at least 37 distinct, nonisomorphic designs. 
(See [13], Appendix C, for a discussion of invariant factors of incidence matrices and the underlying design.)

Among the 37 different invariant factor patterns, there are eight distinct 2-ranks. These eight different 2-ranks are 26, 28, 30,32, 34, 36, 37 and 38.

A homomorphism $f$ from $G$ onto $G^{\prime}$ induces, by linearity, a homomorphism from $\mathbb{Z}[G]$ onto $\mathbb{Z}\left[G^{\prime}\right]$. If the kernel of $f$ is the subgroup $U$, let $T$ be a complete set of distinct representatives of cosets of $U$ and, for $g \in T$, set $t_{g}:=|g U \cap D|$. The multiset $\left\{t_{g}: g \in T\right\}$ is the collection of "intersection numbers" of $D$ with respect to $U$. The image of $\hat{D}$ under the function $f$ is $f(\hat{D})=\sum_{g \in T} t_{g} f(g)$. This group ring element satisfies the equation

$$
f(\hat{D}) f(\hat{D})^{(-1)}=(k-\lambda) 1_{G^{\prime}}+\lambda|U| \hat{G}^{\prime}
$$

in the group ring $\mathbb{Z}\left[G^{\prime}\right]$. The contraction of $D$ to a smaller homomorphic image often provides useful conditions on the existence of a difference set in the original group. Because the size of $D$ is $k$ we have the equation

$$
\sum_{g \in T} t_{g}=k .
$$

Because the coefficient of the identity element is the same on both sides of equation 2 we have the equation

$$
\sum_{g^{\prime} \in T} t_{g}^{2}=(k-\lambda)+\lambda|U| .
$$

For example, suppose $D$ is a $(96,20,4)$ difference set in $G$ and $G^{\prime}$ is a homomorphic image of order 24 . One solution to the equations

$$
\sum_{i=1}^{24} t_{i}=20, \quad \sum_{i=1}^{24} t_{i}{ }^{2}=32
$$

is a list $\left\{t_{i}: 1 \leq i \leq 24\right\}$ with a single 4 , sixteen $1 \mathrm{~s}$, and seven $0 \mathrm{~s}$. When this occurs, one can show that there is a subgroup $H^{\prime}<G^{\prime}$ of order eight such that $f(\hat{D})$ is equivalent to (4) $1_{G^{\prime}}+\left(\hat{G}^{\prime}-\hat{D}^{\prime}\right)$.

The existence of such a homomorphic image gives considerable information about $D$. In this case, if no other information is available, an exhaustive search through the cosets of $U$ where $|D \cap g U|=1$ will require a search space at size at most $4^{16-1} \approx 10^{9}$. Additional information, such as intersections sizes with a subgroup $W$ of size 3, considerably shorten the exhaustive search. (In this work we used both the normal subgroups of orders 3 and 4 to keep the search space relatively small.) Further details on this approach, including GAP programs used, is available in [2] and at the webpage [16].

\section{Summary of past work on $(96,20,4)$ difference sets}

In this paper we will refer to the groups as they appear in the SmallGroups library of the software package GAP (Groups, Algorithms and Programming, [10].) For instance, 
when we work in groups of order 96 and write GAP [96,204] we mean group number 204 of order 96 in the GAP library. (GAP would list such a group as "[96, 204]".)

Before this paper, we had the following results.

McFarland, [14], used vector spaces of dimension $d+1$ over finite fields of order $q$ to construct difference sets with parameters $v=q^{d+1}\left(\frac{q^{d+1}-1}{q-1}+1\right), k=q^{d} \frac{q^{d+1}-1}{q-1}$ and $\lambda=$ $\left.q^{d} \frac{q^{d}-1}{q-1}\right)$ in abelian groups with an elementary abelian subgroup of order $q^{d+1}$. McFarland's construction (with $q=4, d=1$ ) gives $(96,20,4)$ difference sets in $\operatorname{GAP}[96,231] \cong \mathbb{Z}_{2}^{4} \times \mathbb{Z}_{6}$ and $\operatorname{GAP}[96,220] \cong \mathbb{Z}_{2}^{3} \times \mathbb{Z}_{12}$.

Dillon, [7], generalized McFarland's construction to work for a larger set of groups. He constructed McFarland difference sets in groups that have an elementary abelian normal subgroup of order $q^{d+1}$ in its center. Dillon's construction gives $(96,20,4)$ difference sets in GAP $[96,218]$ and GAP $[96,230]$.

Arasu and Sehgal, [4], constructed a $(96,20,4)$ difference set in the abelian group $\operatorname{GAP}[96,161] \cong \mathbb{Z}_{2} \times \mathbb{Z}_{4} \times \mathbb{Z}_{12}$

Golemac, Mandić and Vučičić, [8], constructed $(96,20,4)$ difference sets in five nonabelian groups. These five nonabelian groups are GAP [96,72], GAP [96,78], GAP[96,147], GAP $[96,174]$ and GAP $[96,209]$.

AbuGhneim and Smith, [1, 2], constructed $(96,20,4)$ difference sets in groups that have $\mathbb{Z}_{2}^{4}$ as a normal subgroup. There are 19 groups of order 96 that have $\mathbb{Z}_{2}^{4}$ as a normal subgroup. These groups are GAP $[96, i]$ where $i$ in the set $\{70,159,160,162,167,194$, 195, 196, 197, 218, 219, 220, 221, 226, 227, 228, 229, 230, 231\}.

In recent work, [9], Golemac, Mandić and Vučičić have constructed $(96,20,4)$ difference sets in 22 nonabelian groups. These groups are GAP $[96, i]$ where $i$ in the set $\{13,41,64$, 70, 71, 87, 144, 159, 160, 167, 185, 186, 188, 190, 194, 195, 196, 197, 226, 227, 228, 229\}.

A result of Turyn ([17]) rules out the existence of difference sets in $\operatorname{GAP}[96,2] \cong \mathbb{Z}_{96}$ and $\operatorname{GAP}[96,59] \cong \mathbb{Z}_{2} \times \mathbb{Z}_{48}$.

Arasu, Davis, Jedwab, Ma and McFarland, [3], ruled out the existence of difference sets in the last two abelian groups, $\operatorname{GAP}[96,46] \cong \mathbb{Z}_{4} \times \mathbb{Z}_{24}$ and $\operatorname{GAP}[96,176] \cong \mathbb{Z}_{2} \times \mathbb{Z}_{2} \times \mathbb{Z}_{24}$. As a result of this work, we may conveniently summarize the existence of $(96,20,4)$ difference sets in abelian groups: "An abelian group $G$ has a $(96,20,4)$ difference set if and only if the exponent of $G$ is no larger than 12."

A result of AbuGhneim and Smith, [1, 2], ruled out any group $G$ which has $\mathbb{Z}_{2} \times \mathbb{Z}_{24}$ or $\mathbb{Z}_{2} \times \mathbb{D}_{24}$ or $\left(\mathbb{Z}_{3} \rtimes \mathbb{Z}_{8}\right) \times \mathbb{Z}_{2}$ or $\mathbb{D}_{48}$ as a factor group. Using this result we rule out difference sets in GAP $[96, i]$, where $i$ is in the set $\{6,7,8,9,11,18,19,25,28,37,48,55$, $60,76,80,81,82,89,93,102,104,109,110,111,112,115,116,127,132,134,137,207\}$.

Undergraduate students, Nichols ([15], under the supervision of Harriet Pollatsek, Mt. Holyoke College) Axon, and Gotman, ([5], under the supervision of Emily Moore, Grinnell College) used $(16,6,2)$ difference sets to construct images $(96,20,4)$ difference sets in groups of order 32 and then used those images to construct $(96,20,4)$ difference sets in GAP [96,221] and GAP[96,231]. The difference sets found during this search had the same 2-rank as difference sets which had been previously discovered. 


\section{New Results on the existence of $(96,20,4)$ Differ- ence sets}

Suppose $G$ is a group of order 96 which has normal subgroups of order 3 and 4 and suppose that $D$ is a difference set in $G$. We used GAP to build the 4-images from the 2 -images then again the 8-, 16-, 32-images from the 4-, 8-, 16-images respectively. In a similar way we computed the 24 -images. We wrote programs that combined the 32 - and 24-images to construct difference sets in $G$ or to show such a difference set does not exist after exhaustive search. One can find these programs with examples to explain them in the dissertation of the first author, [2], and on the webpage of the second author, see [16]. We note that these programs give, by exhaustive search, all possible $(96,20,4)$ difference sets in some 72 groups groups of order 96, those groups that have both factor groups of order 32 and 24.

Table 1 lists the Groups of order 96 which admit a $(96,20,4)$ difference sets and have factor groups of orders 32 and 24. In the first column of Table 1 appears the catalogue number $i$ of the group [96,i], according to the GAP SmallGroup library of groups of order 96. (The GAP command "e $:=\operatorname{Elements}(\operatorname{SmallGroup}(96, i))$;" can be used to create a list $e$ of the elements of the group $G$, indexed from 1 to 96.) The indices of the elements of the difference set are provided in the second column of Table 1. The third column provides, in abbreviated form $\left(r_{0}, r_{1}, r_{2}, r_{3}, r_{4}\right)$, the invariant factors of symmetric designs obtained from these difference sets.

In Table 1, we did not include difference sets which were known before. In each group we list difference sets which give clearly different symmetric designs by providing difference sets with distinct invariant factors. A complete list of nonequivalent difference sets can be found in [2] and [16].

Our programs rule out, after an exhaustive search, the existence of difference sets in 85 groups. These groups are GAP $[96, i]$ where $i$ in the set $\{1,4,5,12,15,16,17,21,22$, $23,24,26,27,29,30,31,32,33,34,35,36,38,39,40,42,43,44,45,47,49,50,53,56$, $57,58,61,62,63,100,106,107,108,113,114,117,118,119,120,121,122,123,124,125$, $126,128,138,139,140,148,149,150,153,154,155,156,157,158,163,171,178,179$, $180,181,182,183,184,208,211,213,214,215,216,217,222,224\}$.

By combining our results with the results that have been done before we have 90 groups of order 96 that admit $(96,20,4)$ difference sets. We have 121 groups that do not admit $(96,20,4)$ difference sets. We have 20 groups that are still in doubt. These groups, where the existence of a difference set is still open, are GAP $[96, i]$ where $i$ in the set $\{3,65$, $66,67,68,69,73,74,187,189,191,192,193,198,199,200,201,202,203,204\}$. (None of the remaining open cases has a normal Sylow-3 subgroup. The group GAP [96,204] is the unique group of order 96 without a normal subgroup of order 4 and looks to be a particularly difficult case. The other 19 open groups have normal subgroups of orders 2 and 4 and so an exhaustive search may still be feasible.)

Abelian groups that admit $(96,20,4)$ difference sets have exponent at most 12 . We note that some of the nonabelian groups in which we have constructed $(96,20,4)$ difference sets have exponent 24 . These are cases were the exponent bound for the abelian case is violated 
by the nonabelian case. Groups which have $(96,20,4)$ difference sets and exponent 24 are GAP $[96, i]$ where $i \in\{10,14,20,51,52,54,177\}$.

The invariant factors obtained from the abelian difference sets are $(30,1,34,1,29)$, $(30,2,32,2,29),(32,3,26,3,31),(32,4,24,4,31)$ and $(34,4,20,4,33)$. These invariant factors are from Table 1 and [1]. All difference sets with other invariant factors are genuinely nonabelian.

Table 1. List of $(96,20,4)$ Difference Sets

\begin{tabular}{|c|c|c|}
\hline$i$ & Elements of $D$ & Invariant factors \\
\hline \multirow[t]{2}{*}{$\overline{10}$} & $\begin{array}{l}1,2,3,5,8,9,16,32,37,38,48 \\
53,57,65,66,67,73,74,76,87\end{array}$ & $(34,6,16,6,33)$ \\
\hline & $\begin{array}{l}1,2,3,5,8,9,12,22,27,30,37,38 \\
46,65,68,69,74,86,90,95\end{array}$ & $(36,6,12,6,35)$ \\
\hline \multirow[t]{10}{*}{13} & $\begin{array}{l}1,2,3,4,10,12,24,26,33,38,40 \\
43,46,55,57,67,77,81,82,83\end{array}$ & $(26,6,32,6,25)$ \\
\hline & $\begin{array}{l}1,2,3,4,10,12,19,21,24,26,38,44 \\
45,46,48,60,78,87,90,95\end{array}$ & $(32,2,28,2,31)$ \\
\hline & $\begin{array}{l}1,2,3,4,10,12,14,26,33,50,55,57 \\
68,69,71,73,81,82,83,92\end{array}$ & $(26,10,24,10,25)$ \\
\hline & $\begin{array}{l}1,2,3,4,7,11,27,28,38,44,51,56,57 \\
58,59,65,79,82,85,96\end{array}$ & $(28,2,36,2,27)$ \\
\hline & $\begin{array}{l}1,2,3,4,7,11,14,28,30,44,53,56,57 \\
59,64,65,75,77,80,94\end{array}$ & $(28,6,28,6,27)$ \\
\hline & $\begin{array}{l}1,2,3,4,7,10,22,24,26,27,38,43,51,57 \\
58,69,70,71,73,88\end{array}$ & $(30,6,24,6,29)$ \\
\hline & $\begin{array}{l}1,2,3,4,7,10,12,14,26,37,44,50,60 \\
75,77,78,79,85,92,93\end{array}$ & $(32,0,32,0,31)$ \\
\hline & $\begin{array}{l}1,2,3,4,7,10,12,14,26,37,44,50,60, \\
66,68,75,78,85,87,88\end{array}$ & $(28,4,32,4,27)$ \\
\hline & $\begin{array}{l}1,2,3,4,7,10,12,14,26,37,44,45,47, \\
50,60,72,73,75,78,85\end{array}$ & $(30,2,32,2,29)$ \\
\hline & $\begin{array}{l}1,2,3,4,7,10,12,14,26,37,41,44,50, \\
60,69,75,78,85,86,95\end{array}$ & $(28,0,40,0,27)$ \\
\hline \multirow[t]{3}{*}{14} & $\begin{array}{l}1,2,3,6,8,27,28,36,37,40,43 \\
51,52,53,55,70,75,83,84,93\end{array}$ & $(38,4,12,4,37)$ \\
\hline & $\begin{array}{l}1,2,3,6,8,16,21,27,28,36,46 \\
51,52,53,62,65,75,77,94,96\end{array}$ & $(36,6,12,6,35)$ \\
\hline & $\begin{array}{l}1,2,3,6,8,12,16,28,30,36,43,52, \\
59,60,61,65,70,79,80,81\end{array}$ & $(36,4,16,4,35)$ \\
\hline 20 & $\begin{array}{l}1,2,3,5,7,8,30,31,35,39,46,54 \\
57,65,74,79,90,91,93,96\end{array}$ & $(36,1,22,1,35)$ \\
\hline
\end{tabular}




\begin{tabular}{|c|c|c|}
\hline \multirow[t]{10}{*}{41} & $\begin{array}{l}1,2,3,4,10,14,19,22,27,39,40,52, \\
57,58,66,68,74,76,87,88\end{array}$ & $(32,2,28,2,31)$ \\
\hline & $\begin{array}{l}1,2,3,4,10,14,19,21,44,46,52,61 \\
62,71,73,74,79,86,87,96\end{array}$ & $(26,6,32,6,25)$ \\
\hline & $\begin{array}{l}1,2,3,4,10,14,19,21,44,45,46,48, \\
52,55,68,69,74,82,83,92\end{array}$ & $(28,2,36,2,27)$ \\
\hline & $\begin{array}{l}1,2,3,4,10,12,19,22,24,26,33,38,40, \\
57,67,79,81,82,83,91\end{array}$ & $(30,6,24,6,29)$ \\
\hline & $\begin{array}{l}1,2,3,4,7,10,14,22,43,52,61,62, \\
70,71,73,74,79,86,87,96\end{array}$ & $(26,10,24,10,25)$ \\
\hline & $\begin{array}{l}1,2,3,4,7,10,14,22,43,45,48,52, \\
55,68,69,70,74,82,83,92\end{array}$ & $(28,6,28,6,27)$ \\
\hline & $\begin{array}{l}1,2,3,4,7,10,14,16,30,44,51 \\
52,54,66,67,68,74,81,87,88\end{array}$ & $(30,2,32,2,29)$ \\
\hline & $\begin{array}{l}1,2,3,4,7,10,14,16,30,44,51,52, \\
54,55,67,74,80,81,91,96\end{array}$ & $(28,4,32,4,27)$ \\
\hline & $\begin{array}{l}1,2,3,4,7,10,14,16,30,34,44,51, \\
52,54,62,67,74,81,82,94\end{array}$ & $(32,0,32,0,31)$ \\
\hline & $\begin{array}{l}1,2,3,4,7,10,12,22,24,26,38,43, \\
54,60,70,71,73,76,87,95\end{array}$ & $(28,0,40,0,27)$ \\
\hline \multirow[t]{2}{*}{51} & $\begin{array}{l}1,2,3,4,6,10,13,19,30,32,58,61,62, \\
69,75,77,79,82,88,96\end{array}$ & $(36,6,12,6,35)$ \\
\hline & $\begin{array}{l}1,2,3,4,5,16,18,25,37,58,62,63 \\
64,80,81,86,88,90,91,95\end{array}$ & $(34,6,16,6,33)$ \\
\hline \multirow[t]{3}{*}{52} & $\begin{array}{l}1,2,3,4,8,10,18,19,27,35,41,43, \\
45,48,58,71,82,87,90,96\end{array}$ & $(34,8,12,8,33)$ \\
\hline & $\begin{array}{l}1,2,3,4,8,10,17,18,19,27,41,45, \\
48,64,65,74,83,89,91,94\end{array}$ & $(36,8,8,8,35)$ \\
\hline & $\begin{array}{l}1,2,3,4,5,8,16,19,32,43,46,49, \\
55,58,63,70,73,86,92,94\end{array}$ & $(34,10,8,10,33)$ \\
\hline \multirow[t]{4}{*}{54} & $\begin{array}{l}1,2,3,4,6,12,24,29,38,39,47 \\
50,55,58,60,66,80,82,83,94\end{array}$ & $(36,6,12,6,35)$ \\
\hline & $\begin{array}{l}1,2,3,4,6,11,14,21,23,42,45,50, \\
51,58,65,71,74,75,83,86\end{array}$ & $(34,6,16,6,33)$ \\
\hline & $\begin{array}{l}1,2,3,4,5,21,22,34,35,40,51 \\
63,70,73,76,78,81,82,89,90\end{array}$ & $(34,4,20,4,33)$ \\
\hline & $\begin{array}{l}1,2,3,4,5,8,16,32,37,38,46,50,55 \\
65,66,68,82,83,93,95\end{array}$ & $(34,3,22,3,33)$ \\
\hline \multirow[t]{2}{*}{75} & $\begin{array}{l}1,2,3,4,7,9,21,22,24,25,33,35 \\
41,46,48,53,57,71,87,90\end{array}$ & $(34,4,20,4,33)$ \\
\hline & $\begin{array}{l}1,2,3,4,7,8,13,21,22,41,46,47, \\
56,72,74,80,84,92,94,95\end{array}$ & $(32,8,16,8,31)$ \\
\hline
\end{tabular}




\begin{tabular}{|c|c|c|}
\hline 77 & $\begin{array}{l}1,2,3,4,7,9,16,24,43,45,52,55 \\
63,65,71,72,83,84,89,92\end{array}$ & $(34,4,20,4,33)$ \\
\hline \multirow[t]{4}{*}{78} & $\begin{array}{l}1,2,3,4,7,13,21,22,23,26,27,46, \\
49,56,58,60,68,72,75,95\end{array}$ & $(34,3,22,3,33)$ \\
\hline & $\begin{array}{l}1,2,3,4,7,12,25,37,40,41,43 \\
49,56,58,63,68,69,76,78,88\end{array}$ & $(32,4,24,4,31)$ \\
\hline & $\begin{array}{l}1,2,3,4,7,12,13,26,39,44,49 \\
56,57,66,76,78,85,86,87,95\end{array}$ & $(30,8,20,8,29)$ \\
\hline & $\begin{array}{l}1,2,3,4,7,10,11,12,13,31,34,39, \\
44,54,75,81,82,85,92,93\end{array}$ & $(30,7,22,7,29)$ \\
\hline 79 & $\begin{array}{l}1,2,3,4,7,13,21,22,25,29,46 \\
47,51,53,58,60,69,72,74,86\end{array}$ & $(34,4,20,4,33)$ \\
\hline \multirow[t]{3}{*}{83} & $\begin{array}{l}1,2,3,4,7,13,21,22,25,29,46,48, \\
68,71,74,77,79,83,84,87\end{array}$ & $(34,4,20,4,33)$ \\
\hline & $\begin{array}{l}1,2,3,4,7,12,24,25,29,40,41 \\
57,63,65,68,69,70,75,78,88\end{array}$ & $(32,4,24,4,31)$ \\
\hline & $\begin{array}{l}1,2,3,4,7,10,11,12,31,34,40 \\
51,53,58,63,65,70,82,91,93\end{array}$ & $(32,3,26,3,31)$ \\
\hline \multirow[t]{4}{*}{84} & $\begin{array}{l}1,2,3,4,7,21,22,24,25,36,46 \\
47,55,56,62,69,87,89,94,96\end{array}$ & $(32,8,16,8,31)$ \\
\hline & $\begin{array}{l}1,2,3,4,7,21,22,24,25,29,46,47, \\
55,63,72,83,84,88,92,95\end{array}$ & $(34,4,20,4,33)$ \\
\hline & $\begin{array}{l}1,2,3,4,7,12,24,29,35,40,43 \\
52,53,65,66,81,86,87,90,95\end{array}$ & $(34,2,24,2,33)$ \\
\hline & $\begin{array}{l}1,2,3,4,7,9,12,25,40,50,63,65 \\
66,70,78,81,86,87,90,95\end{array}$ & $(32,2,28,2,31)$ \\
\hline \multirow[t]{4}{*}{85} & $\begin{array}{l}1,2,3,4,7,21,22,26,28,30,33 \\
36,45,46,49,53,69,73,75,95\end{array}$ & $(34,4,20,4,33)$ \\
\hline & $\begin{array}{l}1,2,3,4,7,19,25,32,40,49,54,55 \\
56,58,63,64,76,79,80,93\end{array}$ & $(32,2,28,2,31)$ \\
\hline & $\begin{array}{l}1,2,3,4,7,12,13,26,28,44,53,64, \\
66,67,74,76,81,86,87,95\end{array}$ & $(32,8,16,8,31)$ \\
\hline & $\begin{array}{l}1,2,3,4,7,8,19,29,35,40,41,61 \\
68,69,74,77,85,88,93,94\end{array}$ & $(34,2,24,2,33)$ \\
\hline \multirow[t]{2}{*}{86} & $\begin{array}{l}1,2,3,4,7,21,22,25,41,45,46,48 \\
49,56,59,61,63,77,80,86\end{array}$ & $(34,4,20,4,33)$ \\
\hline & $\begin{array}{l}1,2,3,4,7,16,28,32,35,44,51 \\
52,54,58,59,74,82,83,85,94\end{array}$ & $(32,8,16,8,31)$ \\
\hline \multirow[t]{2}{*}{87} & $\begin{array}{l}1,2,3,4,7,12,24,29,35,40,43,52, \\
53,65,66,81,86,87,90,95\end{array}$ & $(30,2,32,2,29)$ \\
\hline & $\begin{array}{l}1,2,3,4,7,12,24,25,36,44,54,56, \\
57,66,67,75,85,86,87,95\end{array}$ & $(30,8,20,8,29)$ \\
\hline
\end{tabular}




\begin{tabular}{|c|c|c|}
\hline & $\begin{array}{l}1,2,3,4,7,12,24,25,29,40,57,63, \\
65,66,70,75,78,86,87,95\end{array}$ & $(32,4,24,4,31)$ \\
\hline & $\begin{array}{l}1,2,3,4,7,12,24,25,29,40,41,57, \\
63,65,68,69,70,75,78,88\end{array}$ & $(32,2,28,2,31)$ \\
\hline & $\begin{array}{l}1,2,3,4,7,10,11,12,31,34,36,37, \\
39,58,70,78,80,82,90,91\end{array}$ & $(30,7,22,7,29)$ \\
\hline & $\begin{array}{l}1,2,3,4,7,10,11,12,31,34,35,37, \\
40,57,70,78,79,83,90,92\end{array}$ & $(32,3,26,3,31)$ \\
\hline 88 & $\begin{array}{l}1,2,3,4,7,21,22,26,28,32,36,46,49, \\
54,58,69,71,73,87,90\end{array}$ & $(34,2,24,2,33)$ \\
\hline & $\begin{array}{l}1,2,3,4,7,21,22,26,28,32,36,46 \\
48,49,54,71,76,81,87,88\end{array}$ & $(34,3,22,3,33)$ \\
\hline & $\begin{array}{l}1,2,3,4,7,21,22,26,28,32,36,45, \\
46,49,54,69,73,76,81,95\end{array}$ & $(34,4,20,4,33)$ \\
\hline 90 & $\begin{array}{l}1,2,3,4,7,21,22,25,29,36,46,48, \\
49,59,68,77,79,84,87,89\end{array}$ & $\overline{(32,8,16,8,31)}$ \\
\hline & $\begin{array}{l}1,2,3,4,7,19,25,35,40,41,56 \\
61,68,69,74,77,80,83,85,88\end{array}$ & $(32,4,24,4,31)$ \\
\hline & $\begin{array}{l}1,2,3,4,7,19,23,25,35,40,47,48, \\
56,61,73,74,77,80,83,85\end{array}$ & $(32,3,26,3,31)$ \\
\hline & $\begin{array}{l}1,2,3,4,7,16,28,44,45,49,52,61, \\
63,64,71,72,80,83,89,92\end{array}$ & $\overline{(34,4,20,4,33)}$ \\
\hline & $\begin{array}{l}1,2,3,4,7,10,11,12,19,31,40,51, \\
53,58,61,63,64,80,94,96\end{array}$ & $(32,2,28,2,31)$ \\
\hline 91 & $\begin{array}{l}1,2,3,4,7,21,22,25,33,35,46,47, \\
53,56,66,74,75,81,88,89\end{array}$ & $(34,3,22,3,33)$ \\
\hline 92 & $\begin{array}{l}1,2,3,4,7,21,22,25,33,35,46,53, \\
56,68,71,73,74,75,81,87\end{array}$ & $(34,3,22,3,33)$ \\
\hline & $\begin{array}{l}1,2,3,4,7,21,22,25,28,46,47,59, \\
62,63,72,74,77,79,88,95\end{array}$ & $(34,4,20,4,33)$ \\
\hline & $\begin{array}{l}1,2,3,4,7,19,25,27,28,40,55,58, \\
60,63,64,74,76,79,80,93\end{array}$ & $(30,8,20,8,29)$ \\
\hline & $\begin{array}{l}1,2,3,4,7,19,23,25,27,28,40,47, \\
48,58,60,63,64,73,74,76\end{array}$ & $(30,7,22,7,29)$ \\
\hline 94 & $\begin{array}{l}1,2,3,4,7,9,21,22,24,25,35,45, \\
46,47,53,58,60,69,75,87\end{array}$ & $(34,4,20,4,33)$ \\
\hline & $\begin{array}{l}1,2,3,4,7,9,16,25,33,43,45,50, \\
63,65,71,72,75,78,81,89\end{array}$ & $(32,8,16,8,31)$ \\
\hline 95 & $\begin{array}{l}1,2,3,4,7,19,24,26,27,35,53,54, \\
55,56,61,65,67,78,83,92\end{array}$ & $(34,4,20,4,33)$ \\
\hline & $\begin{array}{l}1,2,3,4,7,9,21,22,35,46,48,50, \\
52,59,71,79,84,86,88,96\end{array}$ & $\overline{(32,8,16,8,31)}$ \\
\hline
\end{tabular}




\begin{tabular}{|c|c|c|}
\hline & $\begin{array}{l}1,2,3,4,7,8,19,32,35,40,50,55,56, \\
58,75,78,79,80,85,93\end{array}$ & $(34,3,22,3,33)$ \\
\hline 96 & $\begin{array}{l}1,2,3,4,7,19,25,28,40,41,55,63 \\
64,68,69,74,83,84,88,92\end{array}$ & $(34,4,20,4,33)$ \\
\hline 97 & $\begin{array}{l}1,2,3,4,7,9,16,24,37,45,52,62,63 \\
70,71,72,79,82,89,91\end{array}$ & $(34,4,20,4,33)$ \\
\hline \multirow[t]{3}{*}{98} & $\begin{array}{l}1,2,3,4,7,12,13,26,28,44,53,64 \\
66,67,74,76,81,86,87,95\end{array}$ & $(32,2,28,2,31)$ \\
\hline & $\begin{array}{l}1,2,3,4,7,12,13,26,28,44,45,53,64, \\
67,71,72,74,76,81,89\end{array}$ & $\overline{(32,4,24,4,31)}$ \\
\hline & $\begin{array}{l}1,2,3,4,7,10,11,12,13,31,34,44,54,57, \\
64,67,79,90,92,94\end{array}$ & $\overline{(32,3,26,3,31)}$ \\
\hline \multirow[t]{2}{*}{99} & $\begin{array}{l}1,2,3,4,7,13,19,25,41,50,55,56,62 \\
67,68,69,82,85,88,96\end{array}$ & $(34,4,20,4,33)$ \\
\hline & $\begin{array}{l}1,2,3,4,7,9,16,25,33,37,50,57,59 \\
63,70,78,82,83,90,94\end{array}$ & $(32,8,16,8,31)$ \\
\hline 101 & $\begin{array}{l}1,2,3,4,7,21,22,25,29,36,46,48 \\
49,66,68,71,79,83,84,92\end{array}$ & $(34,4,20,4,33)$ \\
\hline \multirow[t]{2}{*}{103} & $\begin{array}{l}1,2,3,4,7,21,22,26,28,30,33,36,45, \\
46,49,53,69,73,75,95\end{array}$ & $(34,4,20,4,33)$ \\
\hline & $\begin{array}{l}1,2,3,4,7,21,22,25,33,35,46,47 \\
53,56,66,74,75,81,88,89\end{array}$ & $\overline{(34,2,24,2,33)}$ \\
\hline 105 & $\begin{array}{l}1,2,3,4,7,16,28,37,45,49,52,59 \\
62,63,70,71,72,77,79,89\end{array}$ & $(34,4,20,4,33)$ \\
\hline \multirow[t]{5}{*}{129} & $\begin{array}{l}1,2,3,4,7,9,16,32,36,45,49,52 \\
54,58,64,70,71,72,89,90\end{array}$ & $(30,8,20,8,29)$ \\
\hline & $\begin{array}{l}1,2,3,4,7,9,16,23,32,36,47,48,49, \\
52,54,58,64,70,73,90\end{array}$ & $(30,7,22,7,29)$ \\
\hline & $\begin{array}{l}1,2,3,4,7,9,13,21,22,27,32,46,47, \\
52,58,66,72,74,88,90\end{array}$ & $(34,4,20,4,33)$ \\
\hline & $\begin{array}{l}1,2,3,4,7,9,12,13,22,31,32,39,41, \\
48,56,67,71,76,90,95\end{array}$ & $(34,2,24,2,33)$ \\
\hline & $\begin{array}{l}1,2,3,4,7,8,21,22,29,35,41,46 \\
62,66,72,73,74,79,82,96\end{array}$ & $(34,3,22,3,33)$ \\
\hline \multirow[t]{2}{*}{130} & $\begin{array}{l}1,2,3,4,7,9,16,33,35,45,50,52 \\
53,57,65,70,71,72,89,90\end{array}$ & $(32,4,24,4,31)$ \\
\hline & $\begin{array}{l}1,2,3,4,7,9,16,23,32,35,44,47,48, \\
50,52,54,73,75,81,85\end{array}$ & $(32,3,26,3,31)$ \\
\hline \multirow[t]{2}{*}{131} & $\begin{array}{l}1,2,3,4,7,9,21,22,36,46,49,52, \\
55,59,61,68,72,73,87,96\end{array}$ & $(32,2,28,2,31)$ \\
\hline & $\begin{array}{l}1,2,3,4,7,9,21,22,35,46,50,52,59, \\
62,68,71,73,77,87,93\end{array}$ & $(34,4,20,4,33)$ \\
\hline
\end{tabular}




\begin{tabular}{|c|c|c|}
\hline & $\begin{array}{l}1,2,3,4,7,9,16,32,36,49,52,54,58, \\
59,64,70,82,83,90,94\end{array}$ & $\overline{(32,8,16,8,31)}$ \\
\hline \multirow[t]{3}{*}{133} & $\begin{array}{l}1,2,3,4,7,9,16,36,43,45,49,52 \\
55,71,72,83,84,85,89,96\end{array}$ & $(34,4,20,4,33)$ \\
\hline & $\begin{array}{l}1,2,3,4,7,8,21,22,24,28,35,46,47 \\
55,66,69,72,82,84,96\end{array}$ & $(32,8,16,8,31)$ \\
\hline & $\begin{array}{l}1,2,3,4,7,8,16,24,28,30,35,44 \\
59,60,75,78,82,83,85,94\end{array}$ & $(32,2,28,2,31)$ \\
\hline \multirow[t]{2}{*}{135} & $\begin{array}{l}1,2,3,4,7,19,25,40,41,49,56,61, \\
63,64,68,69,80,83,88,92\end{array}$ & $(32,2,28,2,31)$ \\
\hline & $\begin{array}{l}1,2,3,4,7,19,24,29,35,40,52,54,55 \\
57,60,75,79,80,85,93\end{array}$ & $(34,4,20,4,33)$ \\
\hline \multirow[t]{2}{*}{136} & $\begin{array}{l}1,2,3,4,7,21,22,24,25,32,36,41,46, \\
48,54,56,76,81,89,95\end{array}$ & $(34,4,20,4,33)$ \\
\hline & $\begin{array}{l}1,2,3,4,7,9,21,22,24,25,35,46 \\
59,61,68,72,73,80,86,92\end{array}$ & $\overline{(32,2,28,2,31)}$ \\
\hline \multirow[t]{4}{*}{141} & $\begin{array}{l}1,2,3,4,7,12,24,25,29,37,40,41 \\
43,63,68,69,78,81,88,90\end{array}$ & $(32,2,28,2,31)$ \\
\hline & $\begin{array}{l}1,2,3,4,7,21,22,25,33,35,46,53,56, \\
68,71,73,74,75,81,87\end{array}$ & $(34,4,20,4,33)$ \\
\hline & $\begin{array}{l}1,2,3,4,7,12,13,26,43,49,53,56, \\
58,65,66,67,86,87,90,95\end{array}$ & $(34,3,22,3,33)$ \\
\hline & $\begin{array}{l}1,2,3,4,7,12,13,26,28,39,44,51, \\
66,74,78,81,85,86,87,95\end{array}$ & $\overline{(34,2,24,2,33)}$ \\
\hline \multirow[t]{6}{*}{142} & $\begin{array}{l}1,2,3,4,7,19,24,25,29,32,40,41, \\
54,63,64,68,69,81,88,90\end{array}$ & $(32,2,28,2,31)$ \\
\hline & $\begin{array}{l}1,2,3,4,7,19,23,24,25,29,32,40,47, \\
48,54,63,64,73,81,90\end{array}$ & $\overline{(32,3,26,3,31)}$ \\
\hline & $\begin{array}{l}1,2,3,4,7,13,21,22,24,25,28,41,46, \\
48,55,71,82,84,92,95\end{array}$ & $\overline{(34,4,20,4,33)}$ \\
\hline & $\begin{array}{l}1,2,3,4,7,10,11,12,19,31,40,51 \\
53,58,61,63,64,80,94,96\end{array}$ & $(32,4,24,4,31)$ \\
\hline & $\begin{array}{l}1,2,3,4,7,9,21,22,24,25,35,46,59 \\
62,68,71,73,79,87,92\end{array}$ & $(34,2,24,2,33)$ \\
\hline & $\begin{array}{l}1,2,3,4,7,9,21,22,24,25,35,46,47 \\
59,62,66,79,88,89,92\end{array}$ & $\overline{(32,8,16,8,31)}$ \\
\hline \multirow[t]{3}{*}{143} & $\begin{array}{l}1,2,3,4,7,8,19,28,36,39,41,68,69 \\
74,80,83,84,85,88,91\end{array}$ & $(30,8,20,8,29)$ \\
\hline & $\begin{array}{l}1,2,3,4,7,8,19,23,28,36,39,47 \\
48,73,74,80,83,84,85,91\end{array}$ & $(30,7,22,7,29)$ \\
\hline & $\begin{array}{l}1,2,3,4,7,8,16,24,29,30,36,43, \\
59,60,76,78,82,83,85,94\end{array}$ & $(34,4,20,4,33)$ \\
\hline
\end{tabular}




\begin{tabular}{|c|c|c|}
\hline \multirow[t]{2}{*}{144} & $\begin{array}{l}1,2,3,4,7,10,11,12,19,31,40,51, \\
53,58,61,63,64,80,94,96\end{array}$ & $(30,2,32,2,29)$ \\
\hline & $\begin{array}{l}1,2,3,4,7,9,21,22,35,46,50,52,59, \\
62,68,71,73,77,87,93\end{array}$ & $(34,2,24,2,33)$ \\
\hline 145 & $\begin{array}{l}1,2,3,4,7,9,16,32,35,44,50,52,54, \\
59,75,81,82,83,85,94\end{array}$ & $(34,4,20,4,33)$ \\
\hline \multirow[t]{4}{*}{146} & $\begin{array}{l}1,2,3,4,7,21,22,25,46,47,49,56,62, \\
63,79,87,88,89,94,96\end{array}$ & $(34,3,22,3,33)$ \\
\hline & $\begin{array}{l}1,2,3,4,7,21,22,24,25,36,46,56 \\
59,62,68,72,73,79,87,91\end{array}$ & $(34,4,20,4,33)$ \\
\hline & $\begin{array}{l}1,2,3,4,7,12,25,37,40,43,49,56,58, \\
63,66,76,78,86,87,95\end{array}$ & $(32,4,24,4,31)$ \\
\hline & $\begin{array}{l}1,2,3,4,7,10,11,12,31,34,37,40 \\
43,53,63,75,81,83,92,93\end{array}$ & $(32,3,26,3,31)$ \\
\hline 147 & $\begin{array}{l}1,2,3,4,7,9,21,22,24,25,35,46,47, \\
59,62,66,79,88,89,92\end{array}$ & $(32,4,24,4,31)$ \\
\hline \multirow[t]{2}{*}{151} & $\begin{array}{l}1,2,3,4,7,9,21,22,32,35,41 \\
46,47,50,52,58,72,75,78,87\end{array}$ & $(34,4,20,4,33)$ \\
\hline & $\begin{array}{l}1,2,3,4,7,8,19,27,28,39,50,53,54 \\
62,63,65,78,91,93,94\end{array}$ & $(34,3,22,3,33)$ \\
\hline \multirow[t]{2}{*}{$\overline{152}$} & $\begin{array}{l}1,2,3,4,7,8,19,24,33,37,53,55 \\
56,57,63,67,75,79,80,93\end{array}$ & $(34,4,20,4,33)$ \\
\hline & $\begin{array}{l}1,2,3,4,7,8,13,19,32,54,55,56,58, \\
67,74,75,79,80,85,93\end{array}$ & $(32,8,16,8,31)$ \\
\hline \multirow[t]{7}{*}{159} & $\begin{array}{l}1,2,3,4,7,11,12,13,21,23,28,53,54, \\
56,60,65,72,85,86,88\end{array}$ & $(32,2,28,2,31)$ \\
\hline & $\begin{array}{l}1,2,3,4,7,9,16,32,36,45,49,52 \\
54,58,64,70,71,72,89,90\end{array}$ & $(32,4,24,4,31)$ \\
\hline & $\begin{array}{l}1,2,3,4,7,9,16,26,43,45,49,53,58, \\
60,63,65,71,72,89,90\end{array}$ & $(30,8,20,8,29)$ \\
\hline & $\begin{array}{l}1,2,3,4,7,9,16,26,32,44,45,49,63, \\
64,71,72,76,78,81,89\end{array}$ & $(30,2,32,2,29)$ \\
\hline & $\begin{array}{l}1,2,3,4,7,9,16,23,32,36,47,48 \\
49,52,54,58,64,70,73,90\end{array}$ & $(32,3,26,3,31)$ \\
\hline & $\begin{array}{l}1,2,3,4,7,9,16,23,26,43,47,48,49, \\
53,58,60,63,65,73,90\end{array}$ & $(30,7,22,7,29)$ \\
\hline & $\begin{array}{l}1,2,3,4,7,10,12,13,26,33,39,44,47, \\
48,52,57,66,81,85,95\end{array}$ & $(32,0,32,0,31)$ \\
\hline \multirow[t]{2}{*}{160} & $\begin{array}{l}1,2,3,4,10,12,13,16,22,29,56,60 \\
64,70,75,76,77,82,92,94\end{array}$ & $(26,10,24,10,25)$ \\
\hline & $\begin{array}{l}1,2,3,4,10,12,13,16,22,29,56,59 \\
60,64,70,75,76,83,91,96\end{array}$ & $(26,6,32,6,25)$ \\
\hline
\end{tabular}




\begin{tabular}{|c|c|c|}
\hline & $\begin{array}{l}1,2,3,4,10,12,13,16,21,29,40,41,46, \\
48,56,60,75,76,86,89\end{array}$ & $(32,2,28,2,31)$ \\
\hline & $\begin{array}{l}1,2,3,4,7,12,24,25,36,40,43,56 \\
58,64,66,78,86,87,90,95\end{array}$ & $(30,2,32,2,29)$ \\
\hline & $\begin{array}{l}1,2,3,4,7,10,13,27,29,40,43,56,57, \\
58,77,78,82,85,92,94\end{array}$ & $(28,6,28,6,27)$ \\
\hline & $\begin{array}{l}1,2,3,4,7,10,13,27,29,40,43,56,57, \\
58,59,78,83,85,91,96\end{array}$ & $\overline{(28,2,36,2,27)}$ \\
\hline & $\begin{array}{l}1,2,3,4,7,10,13,22,29,39,55,56 \\
66,67,68,72,73,82,83,93\end{array}$ & $\overline{(30,6,24,6,29)}$ \\
\hline & $\begin{array}{l}1,2,3,4,7,10,13,22,27,29,39,41,48, \\
56,57,58,67,78,86,89\end{array}$ & $\overline{(32,0,32,0,31)}$ \\
\hline & $\begin{array}{l}1,2,3,4,7,10,12,13,29,33,40,43 \\
53,56,59,78,79,80,85,94\end{array}$ & $(28,0,40,0,27)$ \\
\hline & $\begin{array}{l}1,2,3,4,7,10,12,13,29,33,40,43,53, \\
55,56,78,82,83,85,93\end{array}$ & $\overline{(28,4,32,4,27)}$ \\
\hline 161 & $\begin{array}{l}1,2,3,4,5,8,17,29,31,33,37,53 \\
60,61,64,69,76,77,83,84\end{array}$ & $\overline{(32,4,24,4,31)}$ \\
\hline & $\begin{array}{l}1,2,3,4,5,8,21,22,29,37,46,48, \\
52,68,71,74,77,81,84,95\end{array}$ & $(34,4,20,4,33)$ \\
\hline & $\begin{array}{l}1,2,3,4,5,8,17,20,29,33,37,46,47, \\
53,64,69,73,76,77,83\end{array}$ & $\overline{(32,3,26,3,31)}$ \\
\hline 162 & $\begin{array}{l}1,2,3,4,5,10,13,22,29,30,32,34, \\
39,42,67,69,73,76,86,92\end{array}$ & $(32,2,28,2,31)$ \\
\hline & $\begin{array}{l}1,2,3,4,5,10,13,22,29,30,32,34,38, \\
39,47,69,70,76,92,95\end{array}$ & $(32,0,32,0,31)$ \\
\hline & $\begin{array}{l}1,2,3,4,5,8,17,30,32,36,38,54,65 \\
67,68,69,75,77,83,88\end{array}$ & $(30,8,20,8,29)$ \\
\hline & $\begin{array}{l}1,2,3,4,5,8,17,20,30,32,36,46 \\
47,54,65,69,73,75,77,83\end{array}$ & $(30,7,22,7,29)$ \\
\hline 164 & $\begin{array}{l}1,2,3,4,5,10,13,27,39,44,50,57 \\
59,63,76,80,85,86,87,95\end{array}$ & $(34,4,20,4,33)$ \\
\hline & $\begin{array}{l}1,2,3,4,5,8,21,22,30,36,38,42,47, \\
48,60,77,81,91,93,95\end{array}$ & $(32,8,16,8,31)$ \\
\hline 165 & $\begin{array}{l}1,2,3,4,5,9,21,22,27,46,48,50, \\
56,61,66,79,86,88,89,96\end{array}$ & $(34,4,20,4,33)$ \\
\hline 166 & $\begin{array}{l}1,2,3,4,5,8,9,13,21,22,24,48 \\
52,61,63,67,71,73,90,94\end{array}$ & $\overline{(34,4,20,4,33)}$ \\
\hline 167 & $\begin{array}{l}1,2,3,4,10,11,13,17,27,33,39,40 \\
55,57,63,68,70,72,73,83\end{array}$ & $(30,6,24,6,29)$ \\
\hline & $\begin{array}{l}1,2,3,4,10,11,13,17,27,33,39,40,42, \\
47,55,57,72,83,85,88\end{array}$ & $\overline{(32,0,32,0,31)}$ \\
\hline
\end{tabular}




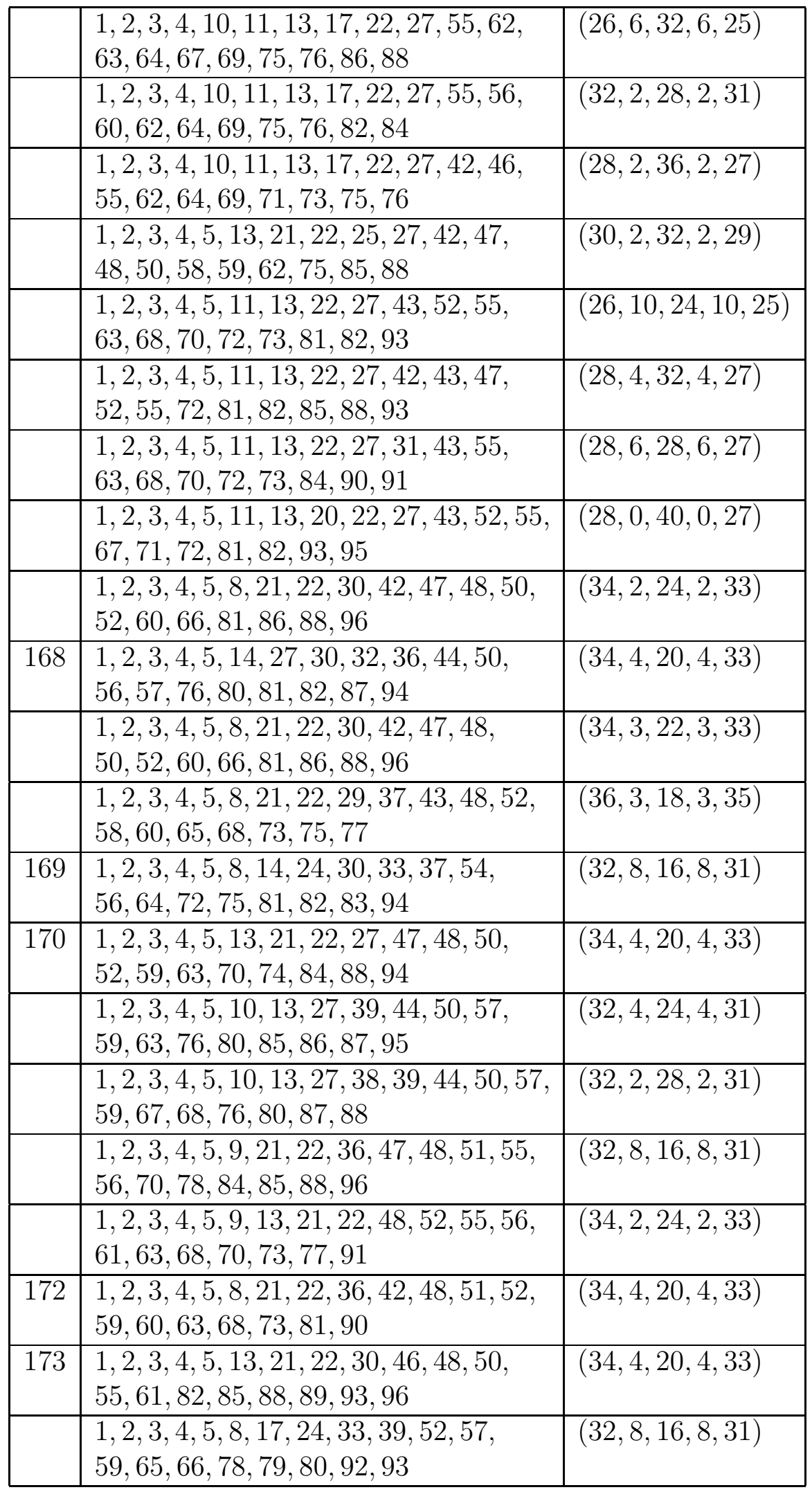




\begin{tabular}{|c|c|c|}
\hline \multirow[t]{3}{*}{174} & $\begin{array}{l}1,2,3,4,5,8,21,22,24,31,42,47,48, \\
59,66,78,81,86,88,96\end{array}$ & $\overline{(32,4,24,4,31)}$ \\
\hline & $\begin{array}{l}1,2,3,4,5,6,7,14,23,28,33,46,51, \\
53,56,72,79,85,87,92\end{array}$ & $(34,4,20,4,33)$ \\
\hline & $\begin{array}{l}1,2,3,4,5,6,7,10,20,23,39,44 \\
50,57,63,76,79,80,87,94\end{array}$ & $(34,1,26,1,33)$ \\
\hline 175 & $\begin{array}{l}1,2,3,4,5,8,9,21,22,37,42,48,51, \\
52,60,68,73,82,90,95\end{array}$ & $(34,4,20,4,33)$ \\
\hline 177 & $\begin{array}{l}1,2,3,5,6,15,17,22,24,26,29,44, \\
56,63,67,71,73,78,79,94\end{array}$ & $(34,7,14,7,33)$ \\
\hline \multirow[t]{3}{*}{205} & $\begin{array}{l}1,2,3,5,7,9,10,22,27,32,37,38,41, \\
48,56,57,65,71,76,86\end{array}$ & $(32,8,16,8,31)$ \\
\hline & $\begin{array}{l}1,2,3,5,7,9,10,14,22,27,28,37,45,57 \\
60,65,68,73,76,87\end{array}$ & $(34,4,20,4,33)$ \\
\hline & $\begin{array}{l}1,2,3,5,7,9,10,12,16,33,38,51,56 \\
64,70,76,80,82,84,92\end{array}$ & $(30,8,20,8,29)$ \\
\hline \multirow[t]{4}{*}{206} & $\begin{array}{l}1,2,3,5,7,9,14,22,27,31,37,41,48, \\
56,57,60,65,76,86,89\end{array}$ & $\overline{(34,2,24,2,33)}$ \\
\hline & $\begin{array}{l}1,2,3,5,7,9,12,14,31,40,43,56,58 \\
68,71,75,78,85,88,89\end{array}$ & $(32,4,24,4,31)$ \\
\hline & $\begin{array}{l}1,2,3,5,7,9,11,12,14,29,40,43,51, \\
61,62,77,78,81,85,96\end{array}$ & $\overline{(32,2,28,2,31)}$ \\
\hline & $\begin{array}{l}1,2,3,5,7,9,11,12,14,29,34,40 \\
43,51,78,81,84,85,91,92\end{array}$ & $(32,3,26,3,31)$ \\
\hline 209 & $\begin{array}{l}1,2,3,6,7,9,10,15,28,33,39,44, \\
47,48,53,57,66,76,85,95\end{array}$ & $(32,4,24,4,31)$ \\
\hline \multirow[t]{2}{*}{210} & $\begin{array}{l}1,2,3,6,7,9,10,15,28,33,39,44,45, \\
53,57,68,69,76,85,89\end{array}$ & $(34,3,22,3,33)$ \\
\hline & $\begin{array}{l}1,2,3,6,7,9,10,15,27,28,32,44,58, \\
64,67,68,69,71,72,90\end{array}$ & $\overline{(34,4,20,4,33)}$ \\
\hline \multirow[t]{4}{*}{212} & $\begin{array}{l}1,2,3,6,7,9,10,15,27,28,30,41,46,47 \\
60,64,65,87,89,90\end{array}$ & $\overline{(30,8,20,8,29)}$ \\
\hline & $\begin{array}{l}1,2,3,6,7,9,10,15,27,28,30,39,47 \\
65,66,69,70,78,81,89\end{array}$ & $(34,4,20,4,33)$ \\
\hline & $\begin{array}{l}1,2,3,6,7,9,10,15,27,28,30,32,46, \\
47,64,65,66,69,75,89\end{array}$ & $(32,8,16,8,31)$ \\
\hline & $\begin{array}{l}1,2,3,6,7,9,10,12,15,23,28,37,46, \\
53,72,76,81,85,86,88\end{array}$ & $(30,7,22,7,29)$ \\
\hline \multirow[t]{2}{*}{218} & $\begin{array}{l}1,2,3,6,7,9,10,12,15,19,32,39,51, \\
56,69,72,73,85,86,90\end{array}$ & $(32,4,24,4,31)$ \\
\hline & $\begin{array}{l}1,2,3,4,7,10,13,26,34,39,44,52, \\
66,68,84,85,87,88,91,92\end{array}$ & $(32,2,28,2,31)$ \\
\hline
\end{tabular}




\begin{tabular}{|c|c|c|}
\hline & $\begin{array}{l}1,2,3,4,7,10,11,13,31,34,39,41, \\
44,48,71,82,85,92,93,95\end{array}$ & $\overline{(32,0,32,0,31)}$ \\
\hline & $\begin{array}{l}1,2,3,4,7,8,10,22,30,32,33,36,41, \\
52,57,64,72,73,85,95\end{array}$ & $(34,2,24,2,33)$ \\
\hline & $\begin{array}{l}1,2,3,4,7,8,10,19,21,23,36,48,52, \\
54,58,60,70,75,86,95\end{array}$ & $(32,3,26,3,31)$ \\
\hline 219 & $\begin{array}{l}1,2,3,5,7,11,14,19,23,28,32,56 \\
67,73,75,78,81,85,86,87\end{array}$ & $(32,0,32,0,31)$ \\
\hline & $\begin{array}{l}1,2,3,4,7,10,12,13,22,26,43,52,53, \\
58,61,62,70,77,90,96\end{array}$ & $(30,4,28,4,29)$ \\
\hline 220 & $\begin{array}{l}1,2,3,4,6,10,11,13,27,38,44,53 \\
56,59,61,66,67,71,72,88\end{array}$ & $(32,2,28,2,31)$ \\
\hline & $\begin{array}{l}1,2,3,4,6,10,11,12,13,32,38,44, \\
45,46,55,69,74,82,86,95\end{array}$ & $(32,0,32,0,31)$ \\
\hline 221 & $\begin{array}{l}1,2,3,4,6,9,16,20,25,26,46,53,58, \\
59,72,74,77,79,82,84\end{array}$ & $(30,4,28,4,29)$ \\
\hline & $\begin{array}{l}1,2,3,4,6,9,16,20,22,25,26,53,59, \\
62,74,77,79,81,82,89\end{array}$ & $(32,0,32,0,31)$ \\
\hline & $\begin{array}{l}1,2,3,4,6,9,14,15,22,31,34,37,41, \\
47,64,70,74,83,84,91\end{array}$ & $(30,1,34,1,29)$ \\
\hline & $\begin{array}{l}1,2,3,4,6,8,10,18,34,39,51,52,54, \\
61,63,66,80,90,91,96\end{array}$ & $(32,4,24,4,31)$ \\
\hline & $\begin{array}{l}1,2,3,4,6,8,10,18,22,34,39,48,51, \\
52,61,63,66,70,89,91\end{array}$ & $\overline{(32,3,26,3,31)}$ \\
\hline 223 & $\begin{array}{l}1,2,3,4,6,9,13,20,23,25,46,47,49, \\
54,58,65,72,84,88,96\end{array}$ & $(34,4,20,4,33)$ \\
\hline 225 & $\begin{array}{l}1,2,3,4,5,6,11,13,19,28,58,63,69, \\
73,83,85,87,91,94,96\end{array}$ & $(34,6,16,6,33)$ \\
\hline & $\begin{array}{l}1,2,3,4,6,8,10,12,15,17,31,36,42,47, \\
48,66,89,91,92,93\end{array}$ & $(37,6,10,6,36)$ \\
\hline & $\begin{array}{l}1,2,3,4,5,13,15,19,20,28,29,36,48, \\
56,59,62,74,85,86,96\end{array}$ & $(36,6,12,6,35)$ \\
\hline 230 & $\begin{array}{l}1,2,3,4,7,10,11,13,31,34,39,41 \\
44,72,73,80,85,86,91,94\end{array}$ & $(30,2,32,2,29)$ \\
\hline & $\begin{array}{l}1,2,3,4,7,10,11,13,27,31,32,40,43, \\
58,61,77,80,85,90,94\end{array}$ & $\overline{(30,1,34,1,29)}$ \\
\hline 231 & $\begin{array}{l}1,2,3,4,5,6,8,12,30,39,42,44,54, \\
55,67,70,72,75,84,86\end{array}$ & $\overline{(30,2,32,2,29)}$ \\
\hline & $\begin{array}{l}1,2,3,4,5,6,8,12,19,32,34,40,42, \\
46,65,72,75,86,90,93\end{array}$ & $(30,1,34,1,29)$ \\
\hline
\end{tabular}




\section{References}

[1] AbuGhneim, Omar A., On nonabelian McFarland difference sets, Proceedings of the Thirty-Fifth Southeastern International Conference on Combinatorics, Graph Theory and Computing., Congr. Numer, 168: 159-175, 2004.

[2] AbuGhneim, Omar A., Nonabelian McFarland and Menon-Hadamard difference sets, Doctoral Diseertation, Central Michigan University, 2005.

[3] K.T. Arasu, J.A. Davis, J. Jedwab, S.L. Ma, and R. McFarland, Exponent bounds for a family of abelian difference sets, Groups, Difference Sets, and the Monster, (Eds. K.T.Arasu, J.F.Dillon, K.Harada, S.K.Sehgal, R.L.Solomon), 145-156, de Gruyter, Berlin-New York, 1996.

[4] K.T. Arasu and S. K. Seghal, Some new difference sets, J. Comb. Th. Ser A, 69: 170-172, 1995.

[5] L. Axon, N. Gotman, Searching for $(96,20,4)$ difference sets, preprint, 2002.

[6] T. Beth, D. Jungnickels, and H. Lenz, "Design Theory", Cambridge Univ. Press, Cambridge 1986.

[7] J. F. Dillon, Variations on a scheme of McFarland for noncyclic difference sets, J. Comb. Th. Ser A, 40: 9-21, 1985.

[8] A. Golemac, J. Mandić and T. Vučičićc, One (96, 20,4)-symmetric design and related nonabelian difference sets, preprint.

[9] A. Golemac, J. Mandić and T. Vučičić, On the existence of difference sets in groups of order 96, preprint.

[10] GAP - Groups, Algorithms and Programming. Version 4.3, The GAP group, Aachen, St. Andrews, 2002. (http://turnbull.mcs.st-and.ac.uk/ gap/)

[11] Yury J. Ionin and Mohan S. Shrikhande, Combinatorics of Symmetric Designs, New Mathematical Monographs, Cambridge University Press, U.K., 2006.

[12] D. Jungnickel, Difference sets, in "Contemporary Desion Theory" (J. H. Dinitz and D. R. Stinson, Eds.) pp. 241-324, Wiley, New York, 1992.

[13] E. S. Lander, "Symmetric Designs: An Algebraic Approach", Cambridge Univ. Press, Cambridge 1983.

[14] R. L. McFarland, A family of difference sets in noncyclic groups, J. Comb. Th. Ser A, 15: 1-10, 1973.

[15] J.P. Nichols, Search for a $(288,42,6)$ difference set, senior thesis, Mount Holyoke College, 2000.

[16] K. Smith difference sets webpage at http://www.cst.cmich.edu/users/smith1kw/ MathResearch/DifferenceSets/DifSets.htm

[17] R. J. Turyn, Characters sums and difference sets, Pac. J. Math., 15: 319-346, 1965. 